\title{
A relationship model for factors influencing knowledge transfer between NPD teams
}

\author{
Alejandro Germán Frank ${ }^{\mathrm{a} *}$, Néstor Fabián Ayala ${ }^{\mathrm{a}}$, Mariano Corso ${ }^{\mathrm{b}}$, José Luis Duarte Ribeiro ${ }^{\mathrm{c}}$ \\ aUniversidade Federal do Rio Grande do Sul, Departamento de Engenharia de Produção e Transportes, \\ Núcleo de Engenharia Organizacional, Porto Alegre, RS, Brasil

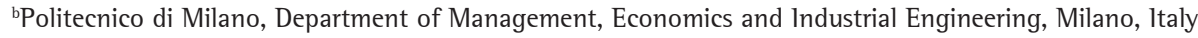 \\ 'Universidade Federal do Rio Grande do Sul, Departamento de Engenharia de Produção e Transportes, Porto Alegre, RS, Brasil \\ *frank@producao.ufrgs.br
}

\begin{abstract}
Paper aims: To define a model that describes the relationship among the main factors influencing knowledge transfer (KT) between new product development (NPD) teams.

Originality: We offer a systemic vision of the relationship between the KT factors. All main KT factors are analyzed together considering the interdependence among them.
\end{abstract}

Research method: KT factors from the literature were used for the model development, which was accomplished through a quantitative evaluation of scholars and practitioners. To test the model, a practical assessment was conducted in five firms using regression analysis.

Main findings: The model describes the relationships of 15 main factors and it can be used for practical assessments or for future theoretical studies.

Implications for theory and practice: The model can help practitioners to use knowledge of their teams for continuous innovation in NPD. 1t allows to perform diagnoses of the KT factors in the specific context of a given firm.

Keywords

New product development. Knowledge management. Knowledge transfer. Knowledge sharing.

How to cite this article: Frank, A. G., Ayala, N. F., Corso, M., \& Ribeiro, J. L. D. (2018). A relationship model for factors influencing knowledge transfer between NPD teams. Production, 28, e20180055. https://doi.org/10.1590/0103-6513.20180055.

Received: June 26, 2018; Accepted: Nov. 12, 2018.

\section{Introduction}

New Product Development (NPD) is a knowledge-intensive activity that generates tacit and explicit knowledge about product design characteristics, development activities and procedures (Rosell et al., 2017; Jin et al., 2018). Such knowledge can provide a competitive position of the companies in the market since it enhances innovation and continuous improvement (Goffin \& Koners, 2011). In order to take advantage from the knowledge generated during NPD, several scholars have studied the contribution of knowledge management strategies (e.g. Yam \& Chan, 2015; Akroush \& Awwad, 2018; Ayala et al., 2017, 2018). When referring to NPD, one of the focus of knowledge management studies is the use of the generated knowledge to improve future or other concurrent projects. This includes the integration of product knowledge-base from different NPD project teams of the same company. Many authors have called this as the knowledge transfer (KT) process between NPD project teams (Argote \& Ingram, 2000; Frank \& Ribeiro, 2014; Frank et al., 2015).

In the literature, there are several studies that have considered how different organizational characteristics and practices can help to foster KT between NPD project teams (e.g. Liu \& Phillips, 2011; Hong et al., 2017; Chang et al., 2017). Some scholars have analyzed these factors from the technological point of view 
(Alavi \& Leidner, 2001; Choi et al., 2008) while others have considered human and organizational aspects (Chang et al., 2017; Hong et al., 2017). Frank et al. (2015) research summarized several KT factors of the literature in a taxonomy. Such work showed a well establish scope of literature on the main managerial aspects of KT. However, as shown in Frank et al. (2015), the literature on KT factors has addressed them from a positivistic point of view, using a causality perspective on factors and KT performance. In the same line, new research on this issue has maintain this perspective (e.g. Rosell et al., 2017; Jin et al., 2018; Akroush \& Awwad, 2018). Additionally, scholars have focused on some specific KT factors and have analyzed them isolated from the influence of other factors. Therefore, such studies present limitations, because, as demonstrated by Bakker et al. (2011), KT is a complex process always involving configurations of multiple factors and none of them is sufficient by itself. This means that KT factors may present synergistic effects with other related factors (Frank \& Ribeiro, 2014). As a consequence, a complete picture of the KT factors and their relationships is still missing and KT theory still needs to see the 'forest beyond the trees', which means a complete vision of KT factors and not only some of them separately from the others. This would help to understand how the different $\mathrm{KT}$ factors may reinforce to each other in the KT process, which can help to build a more effective knowledge management approach for NPD. Therefore, one research question emerges: Which is the relationship among the several different factors that influence KT between NPD project teams? Thus, in this research question, the systemic approach of relationships among factors is highlighted.

Therefore, the objective of this paper is to understand the existing interactions between different factors that can influence the KT between NPD project team and to propose a theoretical model that describes such behavior. Aiming this, we used a semi-quantitative approach based on the analysis of knowledge management experts followed by an empirical study with data collected at five knowledge-intensive and technology-based industrial firms. The results presented in this paper bring the following major contributions: (a) the proposal of a relationship model for describing the KT influence factors, which is useful first for the theoretical understanding of the KT phenomena in NPD; (b) a validation of the proposal in practical cases, illustrating and discussing the suitability of the model to interpret empirical situations; and (c) a new systemic socio-technical perspective of the KT factors in NPD, which provides the basis for future research on this research gap of the literature.

\section{Influence factors on the knowledge transfer process}

Knowledge is considered a blend of experiences, values, contextual information, and insights acquired during the history of a person or a team (Davenport \& Prusak, 1998). Knowledge is generated by people, being considered as a state of mind (Alavi \& Leidner, 2001). Part of this generated knowledge can be explicited and embedded in documents and in several organizational activities (explicit knowledge), while the other part can be shared only by social interaction (tacit knowledge) (Nonaka, 1994; Zollo \& Winter, 2002). In this sense, KT between NPD project teams can be seen as a process of moving both type of knowledge, tacit and explicit, from a project team (source) to another team (recipient) and its subsequent absorption and reusing in new products and technologies to improve the overall performance of NPD (Frank \& Echeveste, 2012; Szulanski, 2000). From this point of view, knowledge needs to be absorbed and should generate a change in results to be considered transferred (Davenport \& Prusak, 1998). In this conception, knowledge is understood by three complementary perspectives (Alavi \& Leidner, 2001): (i) part of knowledge is a state of knowing and understanding; (ii) part of knowledge can be stored and manipulated as an object; (iii) knowledge can be also seen as a process of applying expertise.

The study of KT has considered different perspectives of this phenomena. One of such perspectives considers the nature of the shared knowledge (Nonaka, 1994; Alavi \& Leidner, 2001). The knowledge can be divided into tacit and explicit. Tacit knowledge is intrinsic to individuals, being hard to express and, therefore, it is transferred mainly by social interaction among parties (Nonaka, 1994). On the other hand, explicit knowledge is the part of knowledge that can be established in manuals, business rules and other formal sources, and can be transferred by structural channels (Nonaka, 1994). In our study we consider both type of knowledge. We consider social and technical factors that influence tacit and explicit knowledge respectively. Another stream of research has been dedicated to understand the KT structure, considering the characteristics of source and recipients (Argote \& Ingram, 2000). Source and recipients can be represented by different individuals, teams, functional areas, business units or organizations. Each of them will have different level of knowledge abstraction and representation (Ayala et al., 2017). We focus on the organizational level by considering knowledge of NPD project teams. A third stream on KT has considered the way knowledge can be transferred or also called KT channels (Davenport \& Prusak, 1998). These channels can be informal or formal, the first happens when teams interact naturally with other teams, while formal happens when structured mechanisms such as formal meetings or information systems are used to this aim. We consider both types of channels, since we study a wide spectrum of KT factors, many of them related to formal and other to informal channels. 
KT between NPD project teams is influenced by various organizational factors which may arise from social and technological characteristics of the NDP activities (Frank \& Echeveste, 2012). Several authors have conducted researches on factors from a perspective that involves simultaneously both types of characteristics, which is called as 'sociotechnical approach' (e.g. Pan \& Scarbrough, 1998; Lin \& Lee, 2006; Choi et al., 2008). We used, as theoretical framework, the sociotechnical study of KT factors conducted by Frank et al. (2015) due to some important reasons. Firstly, this is the broader study up to date conducted on KT factors in NPD teams. Frank et al. (2015) conducted a deep systematic review of the literature to identify all the main KT factor that have been addressed in the literature of NPD. After obtaining more than 100 factors, they performed a structured classification and refinement, reducing conceptual redundancies and grouping them by similarities. This effort ended in 16 main factors that aggregate the many different characteristics cited in the literature. Secondly, Frank et al. (2015) study has addressed KT factors from the two main streams of the knowledge management research, one focused on social aspects and the other on technological aspects. They integrated both in a sociotechnical perspective that provides a wider vision of organizational characteristics that are relevant for KT in NPD. Finally, the resulting organization of factors was validated by Frank et al. (2015) using both a focus group with scholars and a survey with companies, providing a strong classification in the taxonomy. The taxonomy developed by Frank et al. (2015) is presented in Chart 1. It follow a sociotechnical approach which is based on the macroergonomic systems of Hendrick \& Kleiner (2001) and later expanded by Guimarães (2009), divided in four subsystems of classification: (i) Personnel Subsystem; (ii) Technological Subsystem; (iii) Work Design Subsystem; and (iv) External Environment Subsystem. These subsystems are described next.

Chart 1. Taxonomy of the KT influence factors proposed by Frank et al. (2015).

\begin{tabular}{|c|c|c|c|}
\hline SST & Types of Factors & Factors & Scope \\
\hline \multirow{6}{*}{ 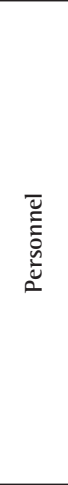 } & \multirow{3}{*}{ Work environment } & Motivation and individual interests (MII) & Willingness to interact and to share knowledge. \\
\hline & & Organizational culture and climate (OCC) & $\begin{array}{l}\text { Disposition of the group to learn, company's traditions, } \\
\text { teamwork style and the feeling of comfort to learn as } \\
\text { a team. }\end{array}$ \\
\hline & & Leadership and organizational strategies (LOS) & $\begin{array}{l}\text { Leaders' encouragement approaches, work style with } \\
\text { the teams and company strategies for human resources } \\
\text { development. }\end{array}$ \\
\hline & \multirow{3}{*}{$\begin{array}{c}\text { Capability } \\
\text { development of staff }\end{array}$} & Human and technical skills (HTS) & $\begin{array}{l}\text { Technical and managerial knowledge and } \\
\text { communicative and cognitive capabilities of the person. }\end{array}$ \\
\hline & & Strategies and practices of team management (SPTM) & $\begin{array}{l}\text { Forms of recruitment, integration and collaborative } \\
\text { team work techniques, incentive methods, knowledge } \\
\text { management activities, etc. }\end{array}$ \\
\hline & & Relationships with R\&D centers (RECE) & $\begin{array}{l}\text { Forms of cooperation and partnership with universities } \\
\text { and R\&D centers through joint research, consulting, } \\
\text { patent transfer, etc. }\end{array}$ \\
\hline \multirow{3}{*}{ 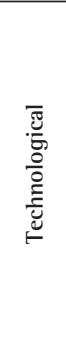 } & \multirow{2}{*}{$\begin{array}{l}\text { Technological } \\
\text { infrastructure }\end{array}$} & IT and integration of databases (1TDB) & $\begin{array}{l}\text { Technologies for managing and storage documents, } \\
\text { for identification of information sources and } \\
\text { communication; level of access allowed to team } \\
\text { members to information and knowledge sources. }\end{array}$ \\
\hline & & Equipment for project development (EQPD) & $\begin{array}{l}\text { Use of laboratory equipment and testing, CAD/CAE } \\
\text { tools, virtual prototypes, and any type of equipment, } \\
\text { whether material or software, which will be useful for } \\
\text { the product development. }\end{array}$ \\
\hline & Physical infrastructure & $\begin{array}{c}\text { Disposition of workplace and adequacy of } \\
\text { infrastructure (WORK) }\end{array}$ & $\begin{array}{l}\text { Layout of the work for team integration. Size of the } \\
\text { work environment and proximity to the equipment } \\
\text { needed. }\end{array}$ \\
\hline \multirow{3}{*}{ 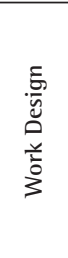 } & \multirow{3}{*}{$\begin{array}{l}\text { Management } \\
\text { practices of product } \\
\text { development }\end{array}$} & Product strategies (PROS) & $\begin{array}{l}\text { Strategic characteristics such as number of projects } \\
\text { to be developed, product innovation efforts, types of } \\
\text { family and product platforms, etc. }\end{array}$ \\
\hline & & Organizational structure and project activities (OSPA) & $\begin{array}{l}\text { Organization of project stages and tasks, use of } \\
\text { management models for project organization, team } \\
\text { arrangements. }\end{array}$ \\
\hline & & Use of methods and tools of NPD (MET) & $\begin{array}{l}\text { Tools used to support product development, such as } \\
\text { QFD, FMEA, Fault Tree, etc. }\end{array}$ \\
\hline \multirow{3}{*}{ 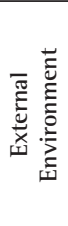 } & \multirow{3}{*}{ Context influence } & $\begin{array}{l}\text { Relationship with suppliers and other companies } \\
\text { (RESU) }\end{array}$ & $\begin{array}{l}\text { Cooperation and partnership with other companies. } \\
\text { Integration with suppliers (co-development). }\end{array}$ \\
\hline & & Government policies (POL) & $\begin{array}{l}\text { Government policies to encourage innovation activities, } \\
\text { partnerships between companies, incentives for } \\
\text { equipment and technology acquisition, etc. }\end{array}$ \\
\hline & & Cultural background of people in the region (CUL) & $\begin{array}{l}\text { Characteristics of the region such as the culture of } \\
\text { people at work, their level of education, etc. }\end{array}$ \\
\hline
\end{tabular}


Personnel Subsystem considers the influence of social elements of the teams. In this sense, people and human aspects are essential elements of the KT process, since KT itself is a process that occurs among people (Edmondson \& Nembhard, 2009). This happens especially in the sharing of tacit knowledge because the interaction between individuals is stronger (Lee \& Choi, 2003).

Technological Subsystem considers the infrastructure of work in the company, such as equipment, tools, automation and physical environment (Hendrick \& Kleiner, 2001). An important aspect that stands out in this subsystem is the use of information systems as way to support KT, especially when the work involves geographically dispersed teams (virtual teams) (Edmondson \& Nembhard, 2009). On the other hand, many companies prefer to work with a traditional approach of co-located teams. In such cases the adequacy of the workplace for interaction among team members is also considered in the Technological Subsystem (Nonaka, 1994; Prencipe \& Tell, 2001).

Work Design Subsystem considers the way in which the company's work was designed (Hendrick \& Kleiner, 2001). In the context of this paper, it concerns the work organization focused on the specific aspects of KT between teams. In this sense, several authors point out that the success of communication and learning between NPD project teams depends on the manner in which the work and structure of the teams are organized (Edmondson \& Nembhard, 2009; Nobeoka \& Cusumano, 1995, 1997). Although the human and technological aspects are relevant to $\mathrm{KT}$, there is also a need of organizing work in such a way that teams can have some interaction that boosts KT. Studies like Nobeoka \& Cusumano $(1995,1997)$ and Aoshima $(2002)$ specifically addressed the adequacy of project structure for this purpose.

External Environment Subsystem includes both other company's processes as well as external factors to the company as the industrial market environment in which the company is located (Du et al., 2007). Therefore, this subsystem delimits the others, demanding that factors from the other subsystems are configured according to it (Hendrick \& Kleiner, 2001).

Considering factors that compose the described subsystems (see Chart 1), this article aims at establishing a model to explain the relationships between them.

\section{Research method}

The model was developed based on a procedure proposed by Saurin et al. (2011). These authors developed a structured analysis of the systemic interaction of Lean practices. As indicated by Saurin et al. (2011), many of these practices do not only affect the Lean operational performance, but they also can have synergistic effects when they are jointly applied. Thus, they developed an assessment procedure based on the opinion of Lean experts to define how these practices interact among them. The procedure assumes as premise that survey methods would be very restrictive in such cases when large number of variables (i.e. Lean practices) need to be evaluated in pairwise comparisons. The application of this procedure follows a mixed method research (Castro et al., 2010). Castro et al. (2010) affirm that mixed methods approaches have more advantages than single qualitative or quantitative approaches, since it offers the descriptive richness of the first and the precision in measurement of the latter. In our study, the collected data is quantitative, based on scores assessment, but the scores were completed and discussed by means of interviews with these experts (qualitative). Validation also follows two stages, first we applied the quantitative assessment in the company and then we discussed it qualitatively with the companies. Thus, we used mixed-methods in this study for 'development purpose' (Greene et al., 1989), which implies a sequential use of single qualitative and quantitative methods (results of one method are used as inputs for the other one).

Summarizing the proposed methodological procedure, it consists in the following stages: (i) construction of the instrument for data collection with NPD experts; (ii) application of the questionnaire with the experts; (iii) analysis of data collected and model construction; and (iv) assessment of the model in real cases. The first three stages allow a deep analysis and the last stage confirms the results using a regression analysis. These stages are explained in the next subsections.

\subsection{Research instrument}

The instrument (questionnaire) was built based on the taxonomy of KT factors presented in Chart 1. As we mentioned in Section 2, Frank et al. (2015) taxonomy is the largest and deepest review on KT factors in NPD project teams available up to date. Therefore, we focus on expanding the understanding of this taxonomy by analyzing the interactions among the KT factors instead of trying to add any other possible factor of other existing studies. 
The questionnaire consists in assessing the strength of relationships among these KT influence factors of Chart 1. Assigned experts should complete the questionnaire by answering the following question: to what extent does factor $X$ contributes to the performance of factor $Y$ ? For this question, the general theoretical importance is assessed and not a particular condition of a company. Considering that 15 factors are analyzed (see Chart 1), $n \times(n-1)$ or 210 assessments were required. The experts were asked to indicate how strong each KT factor interaction is using a five-point discrete scale: 0 (no support/very weak), 1 (weak support), 2 (moderate support), 3 (strong support) and 4 (very strong support).

Once this questionnaire was built, we conducted a pretest based on a practical application. The purpose of this evaluation was to make any necessary adjustment in the questions or factor descriptions. We first tested the understanding of the questions with five scholars of the research team coordinated by the authors of this work and then we reviewed also the questions with a Brazilian company dedicated to hardware development for the telecommunication industry. The company has 650 employees; 310 directly involved in NPD. Nineteen product engineers who occupy different hierarchical levels in the project management structure participated in this pretest. The aim of these tests was to achieve a clear understanding of the explanations regarding each factor for both scholars and practitioners. As result of this stage, some modifications were suggested to clarify the questions and to avoid misunderstandings about the terms used.

\subsection{Application of the questionnaire}

The research method adopted in this work considers situations in which many relationships must be evaluated and cannot be addressed by a traditional survey due to the high amount of data to be collected (Saurin et al., 2011). For such cases, this method focuses on a small number of respondents that must evaluate all possible relationships. The small sample size restriction is compensated by the expertise of the participants, which are chosen in a selective way (only experts of the field) and results are reinforced by practical tests and eventual adjustments, when necessary.

Following such approach, we submitted the final version of the questionnaire to a group of experts in NPD; 15 belong to national and multinational companies operating in Brazil and 7 are scholars from Brazilian major universities who work as researchers in companies. The professional experts were chosen from a professional network of discussion on project and knowledge management practices for NPD in southern Brazil. The whole network is composed by more than 200 companies. We asked to these contacts to provide information of the knowledge management activities developed in the company and then we selected only those companies that have strong activities on knowledge management. We then contacted the main responsible for the knowledge management activities in NPD which was, in most of the cases, the NPD manager or the innovation manager. These practitioners participated in previous research projects between the university and their companies in such issues and, consequently, they have theoretical understanding about the research topic. Scholar experts were selected firstly using the Lattes platform, which is a Brazilian platform were all scholars are registered providing their curriculum vitae. The platform allows to identify experts in different fields. We first searched in this platform those researches with PhD and who work with knowledge management and NPD. Since the list was still large (more than 200 researchers), we only selected those that have been contributors in the proceedings of the biennial conference of the Instituto de Inovação e Gestão de Desenvolvimento do Produto (IGDP), which is the main conference of NPD in Brazil. Then we contacted 20 scholars and obtained the final engagement of 7 experts who participated from our research. Table 1 presents the characteristics of these participants. Contact with the experts was established face-to-face, by e-mail or by telephone and the questionnaire was made available through an address linked to a website. Data inputted by the experts were automatically loaded into a database. Posteriorly, we contacted them again to obtain more information regarding their assessments, in the case where inconsistencies were indentified.

\subsection{Data analysis and construction of the relationship model}

After receiving all participants' answers, we calculated the average scores for each relationship. Following the same methodological procedures used by Saurin et al. (2011) and Frank \& Ribeiro (2014), the relationship model was built considering the relations that show average scores equal to or greater than 2.50 (middle of the five-point scale used). This means that only factors with a moderate to strong influence are considered in the model. The results of this stage were organized in a graphical model by intensity and proximity, allowing the researchers to better analyze the whole scenario and conduct the discussions and interpretations. This stage ended with the construction of a final relationship model for the KT factors. 
Table 1. Characteristics of the experts.

\begin{tabular}{|c|c|c|c|c|c|}
\hline No. & Expert & Position & Industrial Sector & Size of the company" & Size of the NPD team \\
\hline 1 & Professional & Product Engineer & Steel & 5,170 & 43 \\
\hline 2 & Professional & Product Manager & Metallurgical & 3,340 & 109 \\
\hline 3 & Professional & Project Manager & Automotive & 2,760 & 61 \\
\hline 4 & Professional & Product Manager & Automotive & 2,440 & 58 \\
\hline 5 & Professional & R\&D Supervisor & Semiconductor & 1,820 & 35 \\
\hline 6 & Professional & Project Engineer & Automotive & 1,790 & 157 \\
\hline 7 & Professional & R\&D Engineer & Consumer goods & 1,530 & 104 \\
\hline 8 & Professional & R\&D Manager & Industrial equipment & 550 & 21 \\
\hline 9 & Professional & Project Engineer & Computer & 520 & 215 \\
\hline 10 & Professional & Product Engineer & Consumer goods & 480 & 25 \\
\hline 11 & Professional & General Manager & Telecommunications & 410 & 208 \\
\hline 12 & Professional & R\&D Engineer & Electronics & 330 & 32 \\
\hline 13 & Professional & Project Engineer & Engineering Projects & 122 & 15 \\
\hline 14 & Professional & R\&D Engineer & Food & 86 & 11 \\
\hline 15 & Professional & Product Manager & Electronics & 61 & 24 \\
\hline 16 & Academic & Researcher & University - R\&D & -- & -- \\
\hline 17 & Academic & Researcher & University - R\&D & -- & -- \\
\hline 18 & Academic & Researcher & University - R\&D & -- & -- \\
\hline 19 & Academic & Researcher & University - R\&D & -- & -- \\
\hline 20 & Academic & Researcher & University - R\&D & -- & -- \\
\hline 21 & Academic & Researcher & University - R\&D & -- & -- \\
\hline 22 & Academic & Researcher & University - R\&D & -- & -- \\
\hline
\end{tabular}

\subsection{Assessment of the model in real cases}

In the last stage, a quantitative test of the model was carried out based on a study of five companies. The test consists in applying the model for KT diagnosis and comparing results using regression analysis. Companies with different profile were intentional selected aiming at evaluating the model in a diversity of contexts. All these companies have knowledge-intensive activities related to NPD. This means that they develop innovative products; many of them are technology-based products which demand the creation of new knowledge by means of research on new topics; there is a need of multidisciplinary work and of development of new skills and technology competences. Applications were carried out in each company with product engineers, supervisors and managers. Participants from different NPD functions were chosen so that the model could be analyzed through many perspectives. Companies' and participants' profiles are presented in Table 2.

Table 2. Characteristics of the participants of the final test.

\begin{tabular}{clccc}
\hline Case & \multicolumn{1}{c}{ Industrial sector } & Size (employees) & NPD team's size & Interviewees (NPD project team) \\
\hline Company A & Industrial machinery & 500 & 100 & 2 supervisors; 3 product engineers \\
Company B & Agriculture machinery & 2,200 & 150 & 1 supervisor; 1 project leader; 3 product engineers \\
Company C & Oil and Gas equipment & 3,500 & 100 & 1 project manager; 1 supervisor; 3 product engineers \\
Company D & Construction & 4,500 & 50 & 3 supervisors; 2 product engineers \\
Company E & Information technologies & 500 & 200 & 8 project managers; 8 project leaders; 7 product engineers \\
\hline
\end{tabular}

Applications were conducted by individual interviews; each one demanded approximately between one to two hours. During the application, two aspects were evaluated: (i) the support $\left[r_{(F n \rightarrow F)}\right]$ that each factor $\left(F_{n}\right)$ provides to the other factors (i.e. evaluation of the relationships represented in the final model obtained from the experts survey); and (ii) direct evaluation $\left[D A_{F n}\right]$ of the current state of the factor $\left(F_{n}\right)$ (i.e. diagnosis of each factor represented in the final model obtained from the experts survey). Thus, 72 relations and 15 factors (direct evaluation) presented in the model (Figure 1) were analyzed, completing a total of 87 analyzed items. It is important to highlight that the first evaluation criteria enables to assess both perspectives of relationships; i.e. the support $r_{(F n \rightarrow F)}$ that each factor $\left(F_{n}\right)$ provides to others, and the support $r_{(F \rightarrow F n)}$ that each factor $\left(F_{n}\right)$ receives from others. All questions were evaluated by the participants using a 10 point-scale in which 0 represents a condition of no support provided or poor factor performance, while 10 represents an excellent condition of support / performance. After this application, outliers were eliminated and averages were calculated for factors and their relationships in each company according to the following equations: 


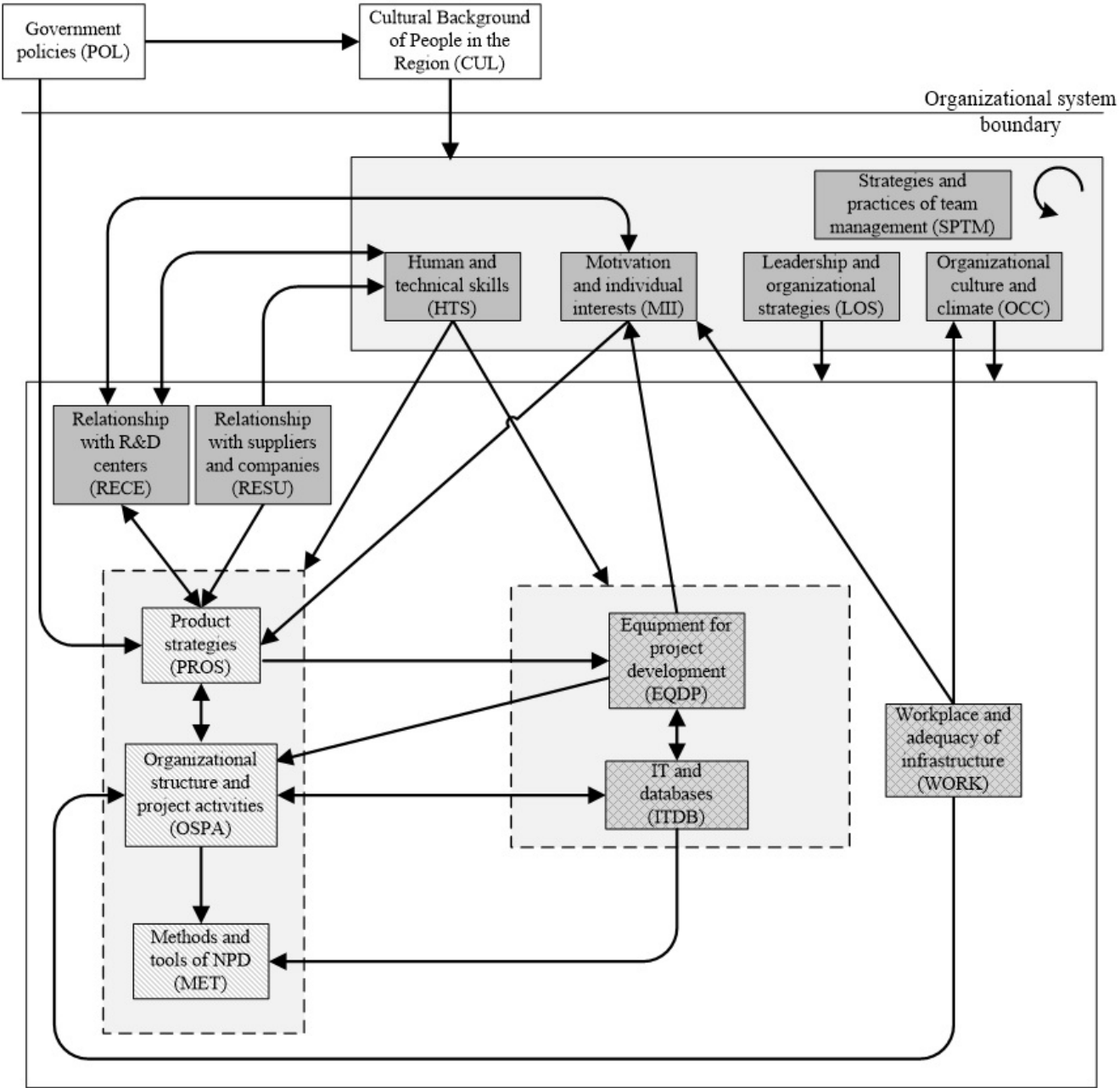

References

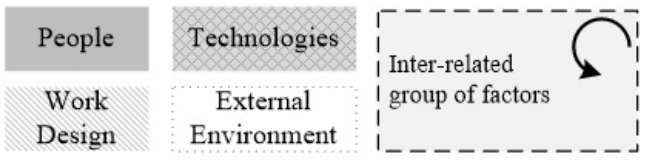

Figure 1. Model of relationships between the inter-project KT influence factors.

$$
\begin{gathered}
R E C_{F n}=\frac{\sum r_{F \rightarrow F n}}{n_{F \rightarrow F n}} \\
\operatorname{PROV}_{F n}=\frac{\sum r_{F n \rightarrow F}}{n_{F n \rightarrow F}}
\end{gathered}
$$

In Equation 1, $R E C_{F n}$ is the average support that factor $F_{n}$ receives from the other factors; $r_{F \rightarrow F n}$ represents the evaluation attributed by the respondents of a company to the support that factor $F_{n}$ receives from the other factors; and $n_{F \rightarrow F n}$ represents the number of factors that provide support to $F_{n}$. On the other hand, $P R O V_{F n}$ is the average support that factor $F_{n}$ provides to the other factors; $r_{F n \rightarrow F}$ represents the evaluation attributed by the respondents of a company to the support that factor $F_{n}$ provides to other factors; and $n_{F n \rightarrow F}$ represents the number of factors that receive support from $F_{n}$. 
In addition, the global performance of factor $F_{n}$ was calculated according to Equation 3:

$$
P E R_{F n}=\frac{P R O V_{F n}+D E_{F n}}{2}
$$

In Equation 3, the global performance of factor $F_{n}\left(P E R_{F n}\right)$ is calculated as the average of two notes: $P R O V_{F n}$ (Equation 2); and the direct evaluation of the current state of the factor $\left(D E_{F n}\right)$ as provided by the respondents. This means that one factor will be considered as having a good performance when it presents a direct good performance $\left(D E_{F n}\right)$ and, at the same time, helps other factors to also achieve good performance $\left(P R O V_{F n}\right)$.

The correspondence of support received (Equation 1) by each of the 15 factors and their respective global performance (Equation 3) was analyzed using linear regression. The hypothesis being tested is that, considering that the proposed model properly represents reality, $R E C_{F n}$ and $P E R_{F n}$ values should present high correlation. Consequently, this validation assumes that factors receiving lower support should also present lower performance, while higher support received should lead to higher performance. Nonetheless, in some particular situations this association might not exist. Positive differences will indicate that performance is superior to the support received. This indicates that the performance level is being sustained by exceptional efforts conducted by the company, which could be reduced through the development of the support factors, so that excessive efforts could be avoided. By the other hand, negative differences will indicate that performance is inferior to the support received. This characterizes an opportunity because conditions to achieve higher performance regarding this specific factor are present, since a reasonable support from other factors is present.

One last point for the model test is that when companies are compared different results are expected. This will indicate that the proposed model has the potential to unveil a diversity of situations according to the specific reality of each case.

\subsection{Validity and reliability of the mixed method research}

As stated by Onwuegbuzie \& Johnson (2006), because mixed methods research consists in combining the strengths of qualitative and quantitative methods without overlapping the weakness of them, assessing the validity of findings involves an integration problem. To assure the validity and reliability of our mixed methods research, we follow Zohrabi's (2013) procedures as explained next.

Regarding the validity of the research, Zohrabi (2013) states that it should be believable and true and it hast to evaluate what it is supposed to evaluate. Thus, the first step is to ensure that the research instrument is valid, reliable and unambiguous. To attend this point, the questionnaire was based on a deep state-of-the-art literature review developed by Frank et al. (2015) to reach the taxonomy of the KT influence factors. Moreover, as explained above, a pretest of the questionnaire was conducted with five scholars and with nineteen product engineers chosen because of their practical experience in the NPD field. This pretest, provided content validity to the research instrument, ensuring the respondents understanding of the questions (Zohrabi, 2013). This means that the interpretation of each factor was discussed and reviewed. The syntaxis and semantics of the explanation of the KT factors was reviewed by them to verify if such interpretation was right. We took notes of their impressions and reviewed them to ensure that the assessment of each KT factor really represents what they are meaning.

To reach internal validity, i.e. the congruence of the research findings with the reality, we use the members' check method and peer examination, as recommended by Zohrabi (2013). Members' check method was applied by presenting to the participant the results and interpretation of the data obtained from the application of the questionnaire (see Section 4.4.). The results were sent by e-mail to each participant asking for feedback if they find any misinterpretation of their opinion. Concerning peer examination process, the research data and findings were reviewed and discussed by three researchers - two are authors of this paper and the third is a research assistant that was included to strength the analysis and interpretations while helping to reduce observers' bias. All these examinations of the findings helped us to assure that they 'make sense' when compared to the companies' reality. In other words, the findings should be aligned to the practitioners experience in their real life at the company.

Furthermore, to increase external validity, i.e. the applicability of the findings in other context or subjects, we carefully selected the professionals and researchers who participated in the research (Zohrabi, 2013). As explained above, they were chosen because of their high expertise in knowledge management practices and NPD. To assure replication of our study (external validation) we described in detail: (i) how the informants were chosen and their characteristics (sections 3.2 and 3.4); (ii) how data was collected (sections 3.2 and 3.4); (iii) how data was analyzed (sections 3.3 and 3.4) and (iv) the analytic constructs and premises (section 4). 


\section{Results}

\subsection{Relationship model between KT influence factors}

Table 3 presents the averages of the relationship intensity between KT influence factors. Factors with average $\geq 2.50$ were highlighted. Figure 1 shows a graphical representation of the results described in Table 3 . In this figure, factors are presented organized by proximity, according to the classification of the sociotechnical systems.

In the following two subsections, results presented in Table 3 and Figure 1 are explained and discussed. First, a detailed analysis of the relationships considered in the model is presented in Section 4.2. This analysis is made by focusing on each subsystem to understand what was stressed in terms of relationships among factors. Next, in Section 4.3, a general overview of the model is presented, in which we summarize the main characteristics discussed in the detailed analysis.

Table 3. Averages of relationship intensities between KT factors.

\begin{tabular}{|c|c|c|c|c|c|c|c|c|c|c|c|c|c|c|c|c|}
\hline & \multicolumn{15}{|c|}{ KT factors as independent variables } \\
\hline & & HTS & Mll & OCC & LOS & SPTM & ITDB & EQPD & WORK & PROS & OSPA & MET & RESU & RECE & POL & CUL \\
\hline \multirow{15}{*}{ 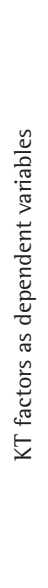 } & HTS & & 3.35 & 3.06 & 3.29 & 3.18 & 2.41 & 2.41 & 1.82 & 2.12 & 2.12 & 2.35 & 2.53 & 2.94 & 1.35 & 2.59 \\
\hline & M11 & 2.65 & & 3.47 & 3.18 & 3.41 & 1.94 & 2.59 & 2.53 & 2.29 & 2.47 & 1.94 & 2.06 & 2.88 & 1.24 & 2.53 \\
\hline & OCC & 3.29 & 2.88 & & 3.59 & 3.06 & 2.12 & 1.65 & 2.59 & 2.00 & 2.25 & 1.76 & 2.00 & 2.35 & 1.41 & 2.65 \\
\hline & LOS & 3.18 & 3.12 & 3.24 & & 2.76 & 1.65 & 1.29 & 1.88 & 2.18 & 1.88 & 1.19 & 1.71 & 2.13 & 1.59 & 2.53 \\
\hline & SPTM & 2.88 & 2.94 & 3.29 & 3.50 & & 1.41 & 1.29 & 2.06 & 1.88 & 1.76 & 1.41 & 1.65 & 2.06 & 1.71 & 2.59 \\
\hline & ITDB & 2.59 & 2.35 & 2.53 & 2.76 & 2.12 & & 2.76 & 2.12 & 2.00 & 2.53 & 2.18 & 1.41 & 1.47 & 1.24 & 1.94 \\
\hline & EQPD & 3.18 & 2.47 & 2.65 & 2.94 & 1.94 & 2.59 & & 2.35 & 2.53 & 2.35 & 2.18 & 1.76 & 2.12 & 1.65 & 1.94 \\
\hline & WORK & 1.88 & 1.82 & 2.76 & 2.82 & 1.59 & 2.00 & 2.06 & & 1.88 & 2.29 & 1.65 & 1.13 & 1.12 & 0.94 & 1.41 \\
\hline & PROS & 3.06 & 2.59 & 3.24 & 3.53 & 2.12 & 2.35 & 2.47 & 1.94 & & 2.94 & 2.47 & 2.53 & 2.71 & 2.53 & 2.24 \\
\hline & OSPA & 3.13 & 2.47 & 2.80 & 3.00 & 2.27 & 2.93 & 2.67 & 2.87 & 2.87 & & 2.27 & 1.80 & 2.13 & 1.00 & 2.13 \\
\hline & MET & 3.13 & 2.33 & 2.87 & 2.93 & 2.13 & 2.53 & 2.07 & 1.53 & 2.47 & 2.80 & & 1.40 & 1.87 & 0.73 & 1.53 \\
\hline & RESU & 2.47 & 2.20 & 2.60 & 2.87 & 1.67 & 1.60 & 1.60 & 0.87 & 2.20 & 1.93 & 1.60 & & 2.00 & 1.87 & 1.73 \\
\hline & RECE & 3.00 & 2.53 & 2.87 & 3.27 & 2.00 & 1.67 & 1.93 & 1.20 & 3.00 & 1.67 & 1.53 & 1.93 & & 2.47 & 2.33 \\
\hline & POL & 1.47 & 1.27 & 2.00 & 2.13 & 1.47 & 1.13 & 0.87 & 0.80 & 2.20 & 1.33 & 1.00 & 1.93 & 2.40 & & 2.07 \\
\hline & CUL & 2.20 & 1.87 & 2.13 & 2.47 & 2.33 & 1.00 & 1.07 & 1.00 & 1.60 & 1.07 & 1.00 & 1.67 & 2.33 & 2.67 & \\
\hline
\end{tabular}

\subsection{Analysis of the model subsystems}

\subsubsection{Personnel subsystem}

Figure 1 shows a set of strongly interrelated factors in the Personnel Subsystem (MIl, OCC, LOS, HTS and SPTM). It is observed that this group is shaped and influenced by the cultural background of people in the region (CUL) where the company is settled. In turn, the cultural background (CUL), which is an external factor, relies on different types of government policies (POL). Thus, the model shows that, for example, incentive policies for training of local people, such as investment in good quality education, will have repercussions on the Personnel Subsystem within the company. Moreover, the factor strategies and practices of team management (SPTM) should fill the gap between Personnel Subsystem factors and the cultural background (CUL). This means that, in the team management (SPTM), problems of lack of training or cultural difficulties should be considered to allow the growing of teams' KT capabilities. Thus, team management (SPTM) is a specific factor for the development of factors related to people, not having a direct relationship, but an indirect one, with the other company's subsystems.

Moreover, the interrelationships in this subsystem are synergistic (excepting the relationships with $R \& D$ centers - RECE). This means that in this block it is not possible to establish which factors are antecedent and which are consequent. They are cyclic and influences are in both directions. Taking as an example the leadership and organizational strategies (LOS) factor and the organizational culture and climate (OCC) factor, the model shows that leadership (LOS) helps to establish a suitable culture and climate (OCC) for learning. It also shows that culture and climate (OCC) contributes for leadership (LOS), thus defining the relationship between teams and their leaders. On the other hand, the model highlights the critical importance of all Personnel Subsystem 
factors as a support to the rest of the KT internal factors. This is especially important regarding leadership (LOS) and culture and climate (OCC) factors, which have an influence on all other internal factors. According to the model, when leadership (LOS) and culture and climate (OCC) are not focused on learning, all other factors are at risk of not having success in the support given to KT.

The above described relationships focused on the Personnel subsystem enables to identify a general pattern. This is illustrated in Figure 2A which provides a simplification of the complete mode (Table 3 and Figure 1). In Figure 2A, a path of influences is shown starting from the external environment, in which government policies (POL) and cultural background (CUL) influence on the Personnel Subsystem and this subsystem has a general influence on Work Design and Technological subsystems.

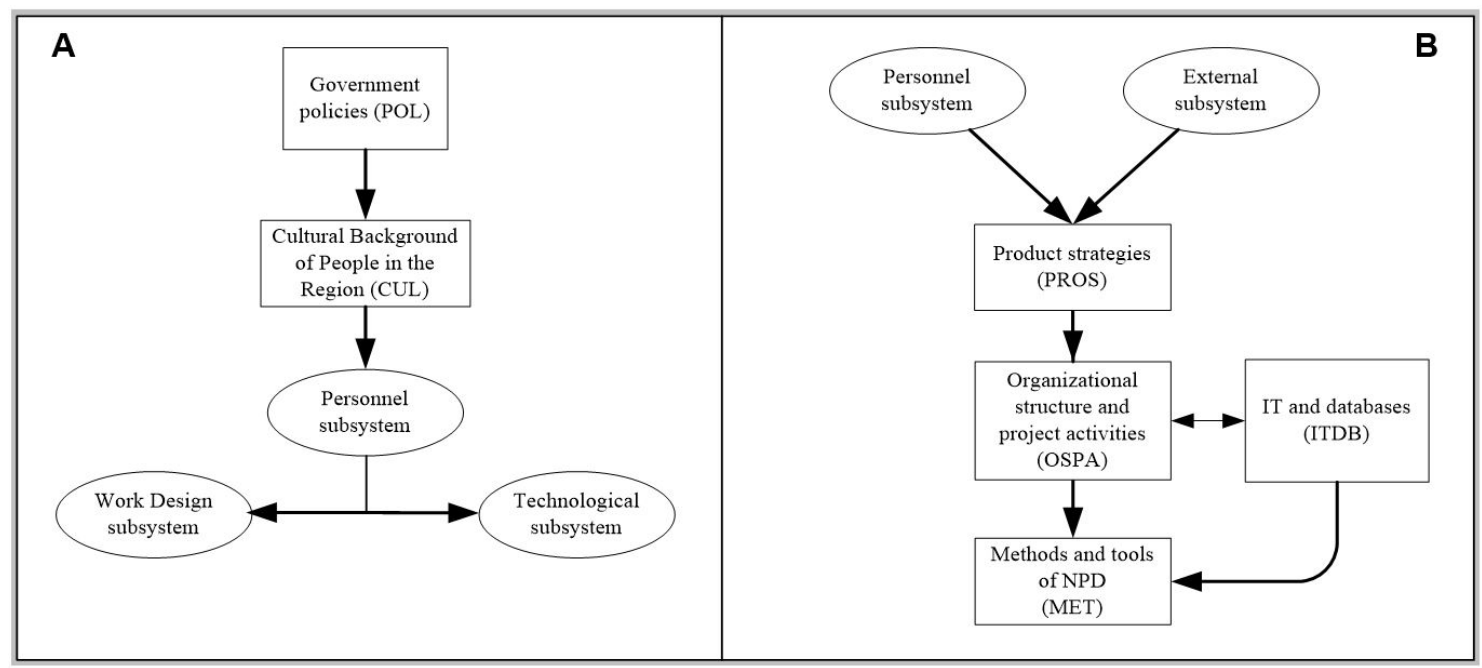

Figure 2. Illustrative simplification of some relationships of the proposed model (Figure 1). (A) Central role of Personnel Subsystem (left side); (B) Relationships between PROS, OSPA and MET (right side).

\subsubsection{Work Design Subsystem}

Considering the Work Design Subsystem (PROS, OSPA and MET), we can see in the general model (Figure 1) that this whole group depends on the human and technical skills (HTS) factor for proper operation, besides depending on leadership and organizational strategies (LOS) and organizational culture and climate (OCC), as mentioned above. It is also observed that government policies (POL), by means of strategies and incentives for innovation and technological development, help to define product strategies (PROS) that the company will adopt. This definition will be crucial to settle the form of organizational structure and project activities (OSPA), which, in turn, will help defining which NPD methods and tools (MET) are the most appropriate ones to be used in the projects. Thus, there is a sequential relationship between the mentioned factors. This is summarized in Figure $2 \mathrm{~B}$, where some relationships of the model have been highlighted. It can be also seen in this figure that IT and databases (ITDB) works as a support for organizational structure and project activities (OSPA) and NPD methods and tools (MET). In this sense, ITDB helps to organize activities of the project (OSPA), while OSPA helps to define the way IT and databases (ITDB) will be used by project teams, contributing to a better use of NPD methods and tools (MET) (e.g. many methods require access to historical data stored in databases). On the other hand, the complete model (Figure 1) shows that there is also a synergistic relationship between product strategies (PROS) and project activities (OSPA), as improvements in OSPA resonate in a positive feedback for PROS.

The Work Design Subsystem is closely related to the other subsystems by means of paths of relationship loops that comprises the product strategies (PROS) factor as pivotal. This can be seen in Figure 3 where some key relationships have been highlighted. Such relationships are described next.

Figure $3 \mathrm{~A}$ shows two paths of influences that start in factor relationship with suppliers and other companies (RESU) and ends in product strategies (PROS). The first path indicates that relationship with suppliers and companies (RESU) helps developing some degree of human and technical skills (HTS) in team members and 


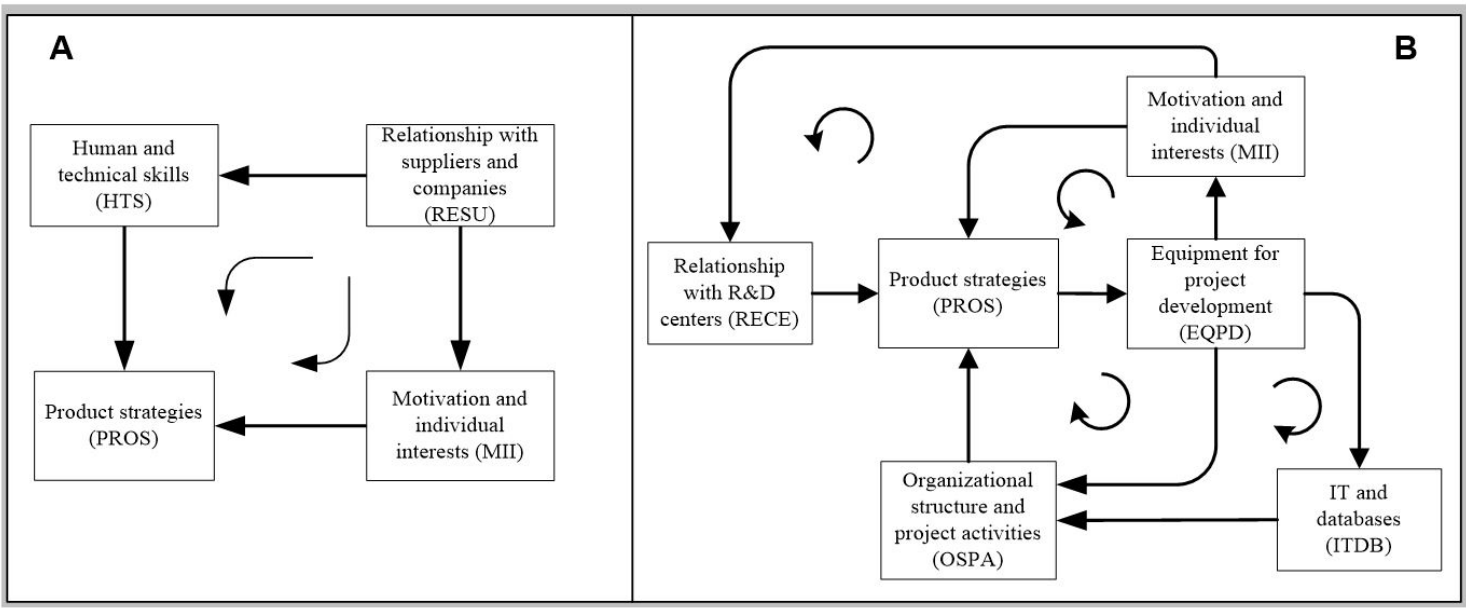

Figure 3. Illustrative simplification of some relationships of the proposed model (Figure 2). (A) Effects on product strategies (left side); (B) Synergistics effects involving product strategies (right side).

this contributes to define better product strategies (PROS). The second path indicates that a good relationship with suppliers and other companies (RESU) improve motivation and individual interests (MII) for transferring knowledge among teams and this also helps to improve product strategies (PROS). Additionally, considering the relationship between product strategies (PROS) and relationship with suppliers and other companies (RESU), the general model of Figure 1 indicates that RESU supports PROS, but the contrary is not noticeable. Consequently, there is a difference between relationship with suppliers and other companies (RESU) and relationship with $R \& D$ centers (RECE) concerning their relationships with product strategies (PROS). Figure 1 shows that, while relationship with $R \& D$ centers (RECE) is directly defined by the company's product strategies (PROS), relationship with suppliers and other companies (RESU) is defined by the leadership and organizational strategies (LOS). This may be due to the fact that relationship with suppliers and other companies (RESU) involves also other processes such as manufacturing and sales. Therefore, leadership and organizational strategies (LOS) helps defining relationship with suppliers (RESU) that, in turn, helps defining forms of product strategies (PROS). For example, leadership and organizational strategies (LOS) can define that the development and manufacturing of some product components will be outsourced, adjusting product strategies (PROS) to such broader strategy.

Figure 3B shows four loops all related to the influence that product strategies (PROS) has on equipment for project development (EQPD). According to Figure 3B, one of these loops shows that product strategies (PROS) help to define investments on new equipment for project development (EQPD). When the EQPD factor is encouraged by product strategies (PROS), it motivates and encourages individuals (MII), resulting in benefits to establish higher challenges in transferring knowledge concerning product innovation and complexity. Such motivation and interests (MII) also reinforces relationships with $R \& D$ centers (RECE). For example, when team members participate in training courses in universities or when they develop their own academic theses with applications in the company, resulting on new benefits for product strategies (PROS).

The second loop of Figure 3B shows that product strategies (PROS) define investments in equipment for project development (EQPD). The availability of appropriate equipment (EQPD) contributes to organize project structure and product development activities (OSPA), which helps readjusting future product strategies (PROS) based on the project structure and activities (OSPA) performance. The third loop is an extension of the second one, since there is only the addition of $I T$ and databases (ITDB) factor as a link between equipment (EQPD) and project structure and product development activities (OSPA). In this sense, EQPD has a direct influence on project activities (OSPA) as shown in the second loop, but in several cases EQPD need also the use of IT and databases (ITDB) (e.g. in the case of virtual simulation equipment or design software such as CAD/CAE) for executing project activities (OSPA), which results in the improvement of product strategies (PROS). Thus, Figure 3B highlight the loop comprising equipment (EQPD) and IT and databases (ITDB). However, the complete model (Figure 1) shows that both factors have also a synergistic relationship, supporting each other and being in many cases close related. The fourth loop of Figure 3B shows that investment in equipment (EQPD) also incentives team motivation and interest (MII) in learning and transferring knowledge, which results in a better performance of product strategies (PROS). 
Another relationship between product strategies (PROS) and relationships with $R \& D$ centers (RECE), not shown in Figure 3B, is expressed in the complete model of Figure 1. This is a synergistic relationship. As Figure 1 indicates, product strategies (PROS) established by the company can consider and encourage forms of partnerships and relationships with $R \& D$ centers, universities or other organizations (RECE). In turn, these relationships (RECE) may result in benefit for products (PROS), developing new strategies based on ideas drawn from the research environment (e.g.: principles and technologies that can be applied to the company's products). Additionally, Figure 1 shows that the incentive for relationships with $R \& D$ centers (RECE) also contributes to the Personnel Subsystem, as it has synergistic relationships with the human and technical skills (HTS) factor and the motivation and individual interests (MII) factor. This is the reason why the relationships with $R \& D$ centers (RECE) has been included in the taxonomy of Frank et al. (2015) within the Personnel Subsystem as a means of encouragement and empowerment of project teams. In this sense, collaborative work with $R \& D$ centers (RECE) helps to bring new knowledge to the teams as well as to develop their technical skills (HTS). On the other hand, technical skills (HTS) of the teams help identifying opportunities for application of academic research from $R \& D$ centers (RECE) in the company's practical problems. Considering the relationships with $R \& D$ centers (RECE), it also provides support for the motivation and individual interests (MII) of people to learn and share knowledge, as it provides them the opportunity to acquire new knowledge and skills in academic environment. On the other hand, motivation and individual interests (MII) are also important for supporting a sustainable relation with $R \& D$ centers (RECE).

\subsubsection{Technological Subsystem}

Regarding the Technological Subsystem, it is observed that human and technical skills (HTS) facilitate two factors in this subsystem: the use of equipment for project development (EQPD) and the use of IT and databases (ITDB) within projects. Considering these two factors, equipment (EQPD) depends on product strategies (PROS), while $I T$ and databases (ITDB) does not, but it depends on the leadership and organizational strategies (LOS) factor. This means that, generally, the leadership (LOS) defines the use of $I T$ and databases (ITDB) and not product strategies (PROS). This is because IT and databases (ITDB) are used at corporate level, while equipment (EQPD) is specific to the development environment. It was also noticed that workplace disposition shared by teams and the adequacy of the infrastructure to the work (WORK) helps developing an organizational culture and climate (OCC) favorable to learning. This helps teams to feel motivated and interested (MII) to share their knowledge through informal conversations and interaction in the workplace. Furthermore, the model shows that workplace disposition (WORK) helps defining organizational structure and project activities (OSPA). This means that project organization will be strongly influenced, and will be configured differently for the work of dispersed or co-located teams. In addition, work layout and infrastructure can help for a better organization of team activities and communication when people with the same interest are close together. Other relationships of this subsystem have been already addressed in the analysis of the Work Design Subsystem, since both groups are closely linked.

\subsubsection{External Environment subsystem}

Finally, considering the External Environment subsystem, the factors government policies (POL) and cultural background (CUL) have a path of influence on the Personnel subsystem as previously showed in Figure 2A. Furthermore, government policies (POL) present a direct influence on product strategies (PROS) (see Figure 1). Such relationships of the External Environment subsystem have been discussed above.

\subsection{General overview of the key-relationships of the model}

Starting from a detailed view of results presented above, in this section we present a resume of the main characteristics of the model, aiming at clarifying the main relationships that were highlighted and presenting a general overview of the key concerns of such model.

First, the proposed model presents blocks of relationships grouped by factors of the same subsystem. This helps to have a better comprehension of the nature of factors that were considered. In Figure $2 \mathrm{~A}$ we presented an abstraction of the model, showing that there is a sequence of main influences between these subsystems. This sequence begins at the External subsystem, which is related to Personnel subsystem and ends up in the influence of Personnel subsystem on two other subsystems: Work Design and Technology. Therefore, the model shows a hierarchical structure of factor relationships, which helps to understand different levels of connection among the elements. 
In Figure 1, we showed that Personnel Subsystem consists in a group of factors completely interrelated. This means that such factors present a synergistic influence (i.e. it is not possible to establish which factors are antecedent or consequent). They support to each other to obtain a suitable performance. Moreover, this subsystem has been shown as crucial to support most of the other internal factors (i.e. factors of Work Design and Technological subsystems).

On the other hand, Work Design did not show synergistic relationships as observed in Personnel subsystem. However, it presents a sequence of influences starting from product strategies and ending in methods and tools (Figure 2B). This shows that, for KT improvement, such sequence of factors relationship should be respected, so that the influence of these factors on NPD and KT could be effective.

Finally, the model has also shown that Product Strategies (PROS) is an essential factor for the systemic structure (Figure 3), since it plays a key role of pivot in the model by articulating the connection among several other factors. Therefore, it is essential to invest efforts in improving this factor first, so that it can support others and KT itself. Other insights that this model helps to unveil have been described in more details in Section 4.2

\subsection{Quantitative test of the final model}

The resulting model from Table 3 and Figure 1 was after tested in five companies. The regression analyses are presented in Figure 4. As shown in this figure, the collected data fitted well to the model. This means that a high positive linear correlation between support received and performance of each factor was obtained in all cases. Moreover, Figure 4 shows that each application presented different results, reflecting the specific situation at each company.
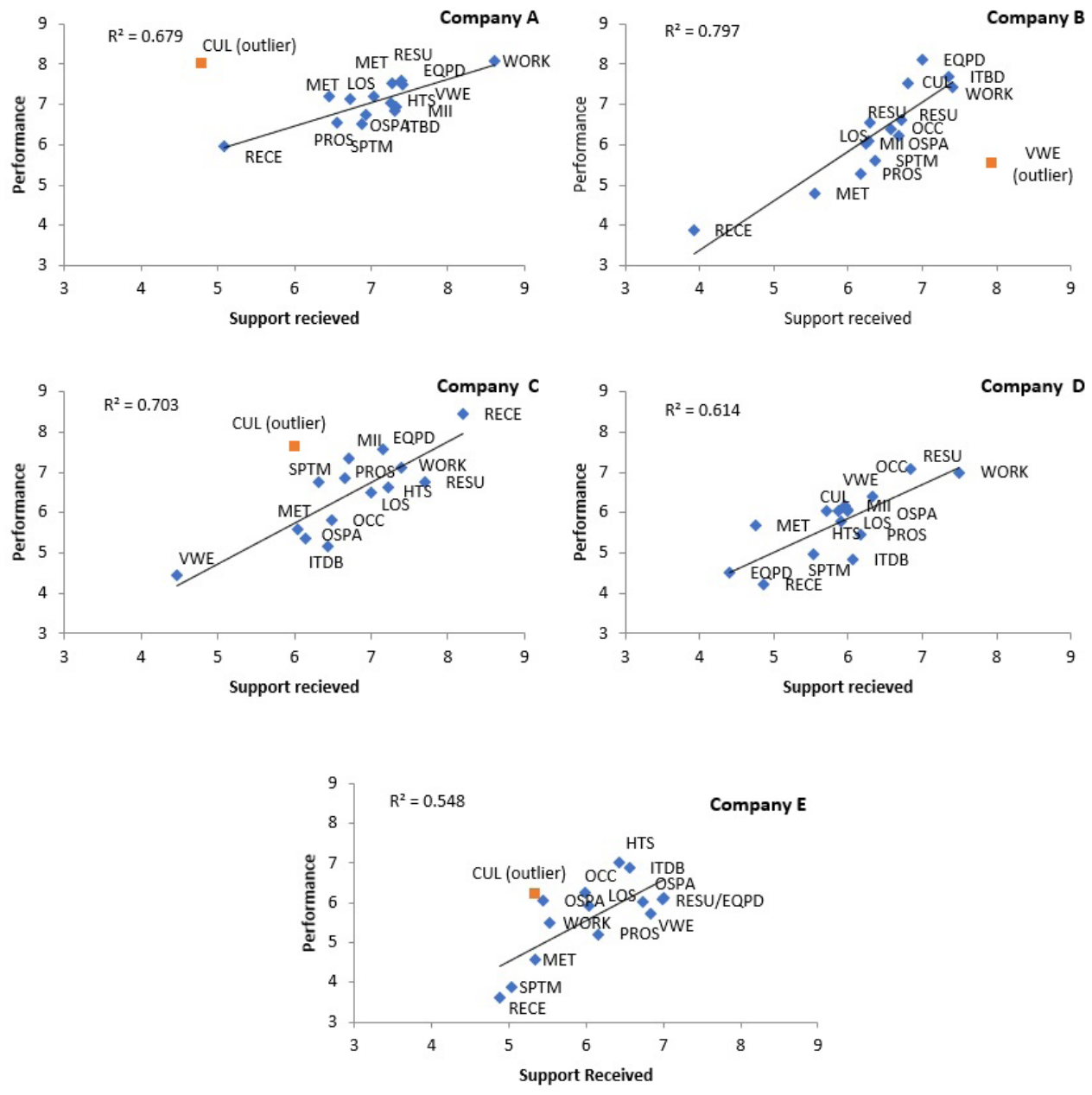

Figure 4. Results of the regression analysis in the study of five companies. 
Results of the regression analysis (Figure 4) also show outliers in Companies A, C and D, always related to the same external factor: cultural background of people in the region (CUL). It should be noted that this is an external industrial market factor, in which companies cannot make a direct influence and, according to the model (Figure 1), it is only influenced by other external KT factor: government policies (POL). In these three cases, interviewees indicated a good performance of factor CUL, even when it is not well supported by factor POL. This condition may be due to the fact that these factors are strongly dependent of temporal and context situations of the industrial market. Factor CUL can present a good performance based on prior government policies, even when current policies are not considered beneficial for industry.

Finally, the quantitative application enabled companies to identify the most important factors to be improved, based on the correlation between support received and performance achieved. Interviewees were able to analyze the critical factors presented in Figure 4 and relate them to the specific support factors that need to be developed. These final results were sent to participants in a report and they confirmed the findings.

\section{Discussions and conclusions}

Our empirical results demonstrated that KT factors cannot be treated simply as direct influencers on KT, since they are interrelated, which results in correlated and synergistic effects. Such findings challenge the conventional wisdom of the field, which has been dedicated only to see KT factors from the point of view of the influence they have on KT, i.e. direct effects (Du et al., 2007; Hong et al., 2017; Chang et al., 2017). Therefore, our work brings KT back to its roots: the field of system dynamics in organizational learning (Argyris, 2017). Authors from that field had proposed that organizations should be considered as a set of dynamic elements interacting and producing conditions to learn and to share knowledge and that no linear effect would be enough to represent such situation of the organizational dynamics (Senge, 1991; Argyris, 2017). Our study redirects the recent research on KT influencing factors to this background, showing that there is complementarity in both fields of knowledge management.

A second important aspect of our findings is that it shows how the external environment has strong implications for internal KT characteristics. Following Frank et al. (2015), we considered government policies and cultural background of the region as factors influencing KT, while most of prior studies did not address so distant factors in the internal KT. However, our model shows that companies cannot think on KT without considering such external conditions. Knowledge is not a physical asset; it is contextual-related and the ways it is transferred depends strongly on external conditions (Malmberg \& Power, 2005). Smeds et al. (2001) and Michailova \& Hutchings (2006) showed how different cultural environments use different ways and approaches for KT. Our work is aligned with such findings, since we show that these external conditions define the personnel subsystem which is over the work and technological subsystems in the KT influence factors.

Third, our model showed the centrality of product strategies in the KT factors (Figure 3B). This means that without having a clear strategy on NPD, which considers product platforms and multiple projects based on such platforms (Gawer \& Cusumano, 2014), it is difficult to target an effective KT between NPD teams. We found that two main pillars support such product strategy, from one hand technical skills of the team and from the other the willingness to establish a good strategy to develop platforms that allow obtaining advantage from the existing knowledge in NPD teams (Figure 3A). The model also shows that these both dimensions are stronger when there is an effective interaction with external suppliers, bringing fresh knowledge from an external source (Ayala et al., 2017, 2018).

\subsection{Limitations and future research}

A first limitation of our work is that we only considered factors included in Frank et al. (2015) prior research. We acknowledge that other factors might exist and are not included here. However, as we explained in our justification of this choice, we understand that the taxonomy used is the broader analysis up to date on KT factors in NPD. Another limitation of this work is that only the relationship among KT factors was analyzed and the KT process itself was not included in such analysis. Hence, once the relationship has been established among the different KT factors, it is still necessary to study how such relationships impact the stages that comprise the KT process in companies. This means that some of the factors considered may be more appropriate, for example, to identify sources of knowledge, while others may be more suitable to record knowledge or for dissemination and absorption of knowledge. Therefore, posterior analysis would also need to consider these differences. Future studies can also advance in this field by using system dynamics as a tool to study the proposed model. In this sense, we advanced the first step by establishing the relationships among the influence factors. System dynamics 
can help to understand how these factors reinforce others and the strength of the outputs. This can be helpful for decision making, so that managers can know what is the expecting performance of the outputs when they give priority to one of the factors that influence others.

\subsection{Managerial implications}

The obtained model can be helpful for practitioners that are concerned with the right use of their team knowledge for continuous improvement and innovation. The proposed model allows to perform diagnoses of the current status of KT factors in the specific context of a given company. So, companies can use this model as a guide to assess KT factors in their NPD environment and process. The model could be applied through focus group discussions or through an internal survey by evaluating team opinion about the real situation of each factor and the support that it receives from other factors. Following this proposal, managers can establish directions for the improvement of KT between NPD project teams. Moreover, our model showed some important aspects managers should care about. Our findings showed that product strategy is a central aspect in the KT factors. KT can be effective only if the organization has a platform strategy with different derivative projects that can take advantage from prior knowledge generated in NPD teams. Therefore, managers should take especial care on this aspect. Our findings also suggest that managers should consider external factors that will define the way knowledge is transferred and the how the other KT factors will behave. In this sense, an effective KT strategy should consider the contextual aspects to define the right KT strategy.

\section{References}

Akroush, M. N., \& Awwad, A. S. (2018). Enablers of NPD financial performance: the roles of NPD capabilities improvement, NPD knowledge sharing and NPD internal learning. International Journal of Quality \& Reliability Management, 35(1), 163-186. http:// dx.doi.org/10.1108/IJQRM-08-2016-0122.

Alavi, M., \& Leidner, D. E. (2001). Knowledge management and knowledge management systems: conceptual foundations and research issues. Management Information Systems Quarterly, 25(1), 107-136. http://dx.doi.org/10.2307/3250961.

Aoshima, Y. (2002). Transfer of system knowledge across generations in new product development: empirical observations from Japanese automobile development. Industrial Relations, 41(4), 605-628. http://dx.doi.org/10.1111/1468-232X.00266.

Argote, L., \& Ingram, P. (2000). Knowledge transfer: a basis for competitive advantage in firms. Organizational Behavior and Human Decision Processes, 82(1), 150-169. http://dx.doi.org/10.1006/obhd.2000.2893.

Argyris, C. (2017). Integrating the individual and the organization. London: Routledge. http://dx.doi.org/10.4324/9780203788417.

Ayala, N. F., Gerstlberger, W., \& Frank, A. G. (2018). Managing servitization in product companies: the moderating role of service suppliers. International Journal of Operations \& Production Management, 1JOPM-08-2017-0484. http://dx.doi.org/10.1108/ 1JOPM-08-2017-0484.

Ayala, N. F., Paslauski, C. A., Ghezzi, A., \& Frank, A. G. (2017). Knowledge sharing dynamics in service suppliers' involvement for servitization of manufacturing companies. International Journal of Production Economics, 193, 538-553. http://dx.doi.org/10.1016/j. ijpe.2017.08.019.

Bakker, R. M., Cambré, B., Korlaar, L., \& Raab, J. (2011). Managing the project learning paradox: a set-theoretic approach toward project knowledge transfer. International Journal of Project Management, 29(5), 494-503. http://dx.doi.org/10.1016/j.jproman.2010.06.002.

Castro, F. G., Kellison, J. G., Boyd, S. J., \& Kopak, A. (2010). A methodology for conducting integrative mixed methods research and data analyses. Journal of Mixed Methods Research, 4(4), 342-360. http://dx.doi.org/10.1177/1558689810382916. PMid:22167325.

Chang, W. J., Liao, S. H., \& Wu, T. T. (2017). Relationships among organizational culture, knowledge sharing, and innovation capability: a case of the automobile industry in Taiwan. Knowledge Management Research and Practice, 15(3), 471-490. http://dx.doi. org/10.1057/s41275-016-0042-6.

Choi, S. Y., Kang, Y. S., \& Lee, H. (2008). The effects of socio-technical enablers on knowledge sharing: an exploratory examination. Journal of Information Science, 34(5), 742-754. http://dx.doi.org/10.1177/0165551507087710.

Davenport, T., \& Prusak, L. (1998). Working knowledge: managing what your organization knows. Boston: Harvard Business School Press.

Du, R., Ai, S., \& Ren, Y. (2007). Relationship between knowledge sharing and performance: a survey in Xi'an, China. Expert Systems with Applications, 32(1), 38-46. http://dx.doi.org/10.1016/j.eswa.2005.11.001.

Edmondson, A. C., \& Nembhard, 1. M. (2009). Product development and learning in project teams: the challenges are the benefits. Journal of Product Innovation Management, 26(2), 123-138. http://dx.doi.org/10.1111/j.1540-5885.2009.00341.x.

Frank, A. G., \& Ribeiro, J. L. D. (2014). Influence factors and process stages of knowledge transfer between NPD teams. International Journal of Quality \& Reliability Management, 31(3), 222-237. http://dx.doi.org/10.1108/1JQRM-04-2012-0046.

Frank, A. G., Ribeiro, J. L. D., \& Echeveste, M. E. (2015). Factors influencing knowledge transfer between NPD teams: a taxonomic analysis based on a sociotechnical approach. $R \& D$ Management, 45(1), 1-22. http://dx.doi.org/10.1111/radm.12046.

Frank, A., \& Echeveste, M. (2012). Knowledge transfer between NPD project teams. International Journal of Quality \& Reliability Management, 29(3), 242-264. http://dx.doi.org/10.1108/02656711211216126.

Gawer, A., \& Cusumano, M. A. (2014). Industry platforms and ecosystem innovation. Journal of Product Innovation Management, 31(3), 417-433. http://dx.doi.org/10.1111/jpim.12105. 
Goffin, K., \& Koners, U. (2011). Tacit knowledge, lessons learnt, and new product development. Journal of Product Innovation Management, 28(2), 300-318. http://dx.doi.org/10.1111/j.1540-5885.2010.00798.x.

Greene, J. C., Caracelli, V. J., \& Graham, W. F. (1989). Toward a conceptual framework for mixed-method evaluation designs. Educational Evaluation and Policy Analysis, 11(3), 255-274. http://dx.doi.org/10.3102/01623737011003255.

Guimarães, L. M. (2009). The practice of ergonomics in the south of Brazil from a sociotechnical perspective. In P. A. Scott (Ed.), Ergonomics in developing regions. Boca Raton: CRC Press.

Hendrick, H. W., \& Kleiner, B. M. (2001). Macroergonomics: an introduction to work system design. Washington: Human Factors and Ergonomics Society.

Hong, J., Lee, 0. K., \& Suh, W. (2017). Creating knowledge within a team: a socio-technical interaction perspective. Knowledge Management Research and Practice, 15(1), 23-33. http://dx.doi.org/10.1057/kmrp.2015.27.

Jin, J. L., Shu, C., \& Zhou, K. Z. (2018). Product newness and product performance in new ventures: contingent roles of market knowledge breadth and tacitness. Industrial Marketing Management. http://dx.doi.org/10.1016/j.indmarman.2018.08.009.

Lee, H., \& Choi, B. (2003). Knowledge management enablers, processes, and organizational performance: an integrative view and empirical examination. Journal of Management Information Systems, 2O(1), 179-228. http://dx.doi.org/10.1080/07421222.2003.11045756.

Lin, H. H., \& Lee, G. G. (2006). Effects of socio-technical factors on organizational intention to encourage knowledge sharing. Management Decision, 44(1), 74-88. http://dx.doi.org/10.1108/00251740610641472.

Liu, Y., \& Phillips, J. S. (2011). Examining the antecedents of knowledge sharing in facilitating team innovativeness from a multilevel perspective. International Journal of Information Management, 31(1), 44-52. http://dx.doi.org/10.1016/j.jinfomgt.2010.05.002.

Malmberg, A., \& Power, D. (2005). (How) do (firms in) clusters create knowledge? Industry and Innovation, 12(4), 409-431. http:// dx.doi.org/10.1080/13662710500381583.

Michailova, S., \& Hutchings, K. (2006). National cultural influences on knowledge sharing: a comparison of China and Russia. Journal of Management Studies, 43(3), 383-405. http://dx.doi.org/10.1111/j.1467-6486.2006.00595.x.

Nobeoka, K., \& Cusumano, M. (1995). Multiproject strategy, design transfer, and project performance: a survey of automobile development projects in the US and Japan. IEEE Transactions on Engineering Management, 42(4), 397-409. http://dx.doi.org/10.1109/17.482089.

Nobeoka, K., \& Cusumano, M. (1997). Multiproject strategy and sales growth: the benefits of rapid design transfer in new product development. Strategic Management Journal, 18(3), 169-186. http://dx.doi.org/10.1002/(SICl) 1097-0266(199703)18:3<169::AIDSMJ863>3.0.CO;2-K.

Nonaka, 1. (1994). A dynamic theory of organizational knowledge creation. Organization Science, 1(5), 14-37. http://dx.doi.org/10.1287/ orsc.5.1.14.

Onwuegbuzie, A. J., \& Johnson, R. B. (2006). The validity issue in mixed research. Research in the Schools, 13(1), 48-63. http://dx.doi. org/10.1016/j.jsis.2005.08.002.

Pan, L. S., \& Scarbrough, H. (1998). A socio-technical view of knowledge-sharing at Buckman Laboratories. Journal of Knowledge Management, 2(1), 55-66. http://dx.doi.org/10.1108/EUM0000000004607.

Prencipe, A., \& Tell, F. (2001). Inter-project learning: Processes and outcomes of knowledge codification in project-based firms. Research Policy, 30(9), 1373-1394. http://dx.doi.org/10.1016/S0048-7333(01)00157-3.

Rosell, D. T., Lakemond, N., \& Melander, L. (2017). Integrating supplier knowledge in new product development projects: decoupled and coupled approaches. Journal of Knowledge Management, 21(5), 1035-1052. http://dx.doi.org/10.1108/JKM-10-2016-0438.

Saurin, T. A., Marodin, G. A., \& Ribeiro, J. L. D. (2011). A framework for assessing the use of lean production practices in manufacturing cells. International Journal of Production Research, 49(11), 3211-3230. http://dx.doi.org/10.1080/00207543.2010.482567.

Senge, P. M. (1991). The fifth discipline, the art and practice of the learning organization. Performance + Instruction, 30(5), 37-37. http://dx.doi.org/10.1002/pfi.4170300510.

Smeds, R., Olivari, P., \& Corso, M. (2001). Continuous learning in global product development: a cross-cultural comparison. International Journal of Technology Management, 22(4), 373-392. http://dx.doi.org/10.1504/1JTM.2001.002970.

Szulanski, G. (2000). The process of knowledge transfer: a diachronic analysis of stickiness. Organizational Behavior and Human Decision Processes, 82(1), 9-27. http://dx.doi.org/10.1006/obhd.2000.2884.

Yam, R. C., \& Chan, C. (2015). Knowledge sharing, commitment and opportunism in new product development. International Journal of Operations \& Production Management, 35(7), 1056-1074. http://dx.doi.org/10.1108/lJOPM-01-2014-0037.

Zohrabi, M. (2013). Mixed method research: instruments, validity, reliability and reporting findings. Theory and Practice in Language Studies, 3(2), 254-262. http://dx.doi.org/10.4304/tpls.3.2.254-262.

Zollo, M., \& Winter, S. G. (2002). Deliberate learning and the evolution of dynamic capabilities. Organization Science, 13(3), 339-351. http://dx.doi.org/10.1287/orsc.13.3.339.2780. 